\title{
Medical Teacher: Monster or Mystery?
}

Bomtaeck Kim

Department of Family Practice and Community Health, Ajou University School of Medicine, Suwon, Korea

\section{의과대학 교수: 불가사리 혹은 불가사의}

아주대학교 의과대학 가정의학교실

\section{김범택}

불가사리라는 전설의 동물이 있다. 불가사리는 곰의 몸과 머 리, 호랑이의 발톱, 코끼리의 코, 무소의 눈을 가졌다고 한다. 작은 밥알 크기의 불가사리는 처음에는 바늘을 먹고 몸이 커 지기 시작하더니 나중에는 쇠로 된 솥이며 농기구를 다 먹어 치웠고 점점 커져서 그 덩치가 기와집만 했다고 한다. 불가사 리(不可殺丹)는 너무나 강해 어떤 창이나 칼로도 해를 입힐 수 없었다. 그러나, 불가사리를 만든 스님이 나타나 그 꼬리에 불 을 놓자 불가사리는 그 동안 먹은 모든 쇠를 내어놓고 다시 밥풀로 돌아갔다고 한다. 이 이야기는 절제되지 않은 인간의 욕망에 대한 은유였다.

의과대학 교수라는 직업은 불가사리다. 옛날의 의학교육은 철저히 교수중심이었다. 의과대학 교수가 하는 교육이면 그 것이 무엇이든 교수가 어떻게 하던 그것이 의학교육이었다. 1990년대부터 의과대학이 기하급수적으로 늘어나면서부터 대학병원도 경쟁을 해야 하는 시대가 왔고, 의과대학 교수는 외래와 수술을 늘려야 했다. 2000년이 되면서부터 의과대학 교수를 하기 위해서는 높은 수준의 논문을 써야 했고 그 기준 은 마치 장대 높이뛰기를 하듯이 점점 높아졌다. 교수의 책임
과 의무는 마치 불가사리가 쇠를 먹고 몸을 키우듯이 늘어났 다. 2000년대 후반부터는 의학교육에서조차 교수의 부담은 늘어나기 시작했다. 강의 평가가 의학대학에도 도입되고 실 기 시험이 시행되었다. 교수들은 강의를 녹화해서 피드백 받 고, 문제바탕학습(problem-based learning, PBL)이나 팀바 탕학습(team-based learning)같은 새로운 교수법을 익혀야 했으며 학생들의 실습을 직접 챙겨야 했고, 임상수행평가 (clinical performance examination)같은 새로운 형태의 시 험에도 적응을 해야 했다. 이런 모든 부담은 교수 업적평가에 반영되어 의과대학 교수들을 짓눌렀다. 그러나 의과대학 교 수들은 이 모든 요구에 훌륭히 부응해 왔다. 늘어나는 환자들 속에서도 환자들의 만족도를 높이기 위해 노력했고 밤에 퇴 근도 못하고 실험실에 앉아 연구를 계속해서 세계적인 잡지 에 논문을 싣고 있고 의학교육을 위해 주말을 희생해서 강의 와 실습, 시험 준비를 해 왔다. 마치 불가사리가 쇠를 먹어 치 우듯이 앞의 주어진 과제를 해결해 왔다. 정말 불가사의(不可 思議한 일이다. Korean Journal of Medical Education 6월 호는 이런 교수들에게 새로운 도전을 던져 주고 있다. 우리는
Received: May 2, 2012 • Revised: May 16, 2012 • Accepted: May 18, 2012 Corresponding Author: Bomtaeck Kim

Department of Family Practice and Community Health, Ajou University School of Medicine, 206 Worldcup-ro, Yeongtong-gu, Suwon 443-721, Korea

Tel: +82.31.219.5309 Fax:+82.31.219.5218 email: lovesong@ajou.ac.kr
Korean J Med Educ 2012 Jun; 24(2): 85-87. http://dx.doi.org/10.3946/kjme.2012.24.2.85 pISSN: 2005-727X eISSN: 2005-7288

(C) The Korean Society of Medical Education. All rights reserved. This is an open-access article distributed under the terms of the Creative Commons Attribution Non-Commercial License (http:// creativecommons.org/licenses/by-nc/3.0/), which permits unrestricted non-commercial use, distribution, and reproduction in any medium, provided the original work is properly cited. 
이제 우리 다음 세대에게 교수의 불가사의한 능력을 물려주 어야 하는 것이다.

첫 번째 도전은 학습 동기 부여이다. 학습이 부진한 학생들 의 학습역량을 강화하는 방법은 여러 가지가 있지만, 어떤 방 법도 학습동기가 결여되어 있으면 무용지물이다. 특히 예과 때를 의사로서의 자질 함양과 의학교육 수학능력 증진의 시간 으로 쓰기보다 동아리와 취미생활로 학습 습관을 잃어버리는 기간이 되고 있는데, 이런 의미로 볼 때 신입생을 대상으로 한 건양대의 동기유발 교육과정은 의의가 크다[1]. 이 교육과정 은 리더십 캠프 등 대학차원의 프로그램부터 전공 맛보기, 의 료인문학 맛보기, 미래의 직장 방문 등 다양한 학습 동기 촉진 자들이 들어 있다. 학습자의 지능이나 적성, 교수의 교수방법 도 학습성과의 개인별 차이를 설명하는 중요한 요소지만 이보 다 더 강력한 요인 중의 하나는 학습자의 동기이며, 교수는 학 생들이 즐거운 마음으로 스스로 학습 행동을 지속할 수 있도 록 도와주어야 한다. 이 연구는 그 비법들을 소개하고 있다.

두 번째 도전은 변화하는 학생이다. 의과대학 학생들은 완 벽주의적·이기적이며, 학업 스트레스가 크고 사회·도덕적 측면에서 도덕판단력은 높은 편이 아니다. 그들은 의사라는 직업이 예전보다 인정받지 못한다는 생각을 하고 있고, 전보 다 더 개방적이고 유연한 의사-환자 관계를 형성하려고 한다 [2]. 생명을 다루고, 환자의 고통과 치유에 정직한 책임을 갖 는 의사들을 양성해야 하는 의학교육에서는 의대생들의 바람 직한 면을 길러주고 그렇지 않은 면을 교정해주는 인문사회 의학적 고려와 지도가 절대 필요하다.

또한 의과대학에서 여학생들의 비율이 증가하면서 이는 의 과대학 나아가 의료사회의 근본적인 변화를 요구하고 있다. 이번 호에 실린 "젠더가 여성수련의의 직업정체성 형성에 미 치는 영향”이란 연구[3]는 젠더라는 렌즈를 하나 더 쓰고, 경 쟁을 통한 수월성 확보를 매우 중요시 하는 의료계에서 경력 개발과정(carrier development)에서 발생할 수 있는 남녀 간 의 불평등에 대해 통렬한 비판을 가하고 있다. 저자는 의료분 야에서 여성의 증가와 지위 향상은 일시적으로 집단 간에 갈 등을 야기할 수도 있으나, 동시에 구조적 자산이 증가한다는 관점에서 접근해야 한다고 주장하고 있다. 이는 젠더적 다양 성을 배척하지 않고 존중하는 조직이 그렇지 않은 조직보다 생산적이고 창의적인 성과 창출이 가능하기 때문이다. 이런
젠더적 관점을 길러주는 것도 의학교육의 몫이 될 것이다. 셋째는 진화하는 교수법이다. 의과대학에서 $\mathrm{PBL}$ 을 한다는 것만으로도 자랑인 시기가 있었다. 그러나, PBL은 전통적 유 형의 시험이나 문제해결능력을 요구하는 시험에서도 우월한 결과를 보이지 못하였다. 이것은 학생들이 논리적 추론을 순 리대로 하지 않고 숙제를 하듯이 기계적으로 서둘러 학습과 제를 잡으려고 하고 자기 성찰을 통한 평가가 필수적이지만 이것이 적절하게 이루어지지 않기 때문인데, 이를 보완하기 위해 PBL은 진화하고 있다는 것을 보여주는 연구가 이번 호 에 수록되었다[4]. 이 연구는 진단 스키마 형, 치료 알고리듬, 사례기전 다이어그램을 PBL에 도입하여 이런 것들을 제작, 활용한 경험을 소개하고 향후 개선 방향을 모색하였다.

이번 호에는 또한 교수들의 재미있는 편견에 대한 고발도 있다. 교수들은 보통 좌석 이동이 없는 학생과 이쪽저쪽으로 자리를 이동해 다니는 학생에 대하여 성적이 차이가 있을 것이 라고 고정관념을 가지고 있지만 한 좌석 구역에 꾸준히 앉는 학생들과 구역 간 좌석 이동을 한 학생 간에 학업성취도 차이 는 없었다[5]. 이는 강의실 좌석은 학생들의 학업성적보다는 교수자의 학생에 대한 편견을 만들어 내는 데 더 공헌을 하고 있다는 것을 보여주는 것이다. 교수는 앞자리에 앉는 학생들이 학습태도가 좋다고 생각할 수는 있지만, 그것을 곧 학업성취도 를 예측하는 잣대로 삼지 않도록 주의해야 할 것이다.

모든 사회가 무한 경쟁에 빠져있지만, 의과대학 교수들만 큼 단기간의 많은 변화를 경험하고 있는 직업도 없는 것 같다. 우리는 의과대학 교수들의 불가사의한 능력이 의학교육을 통 해 다음세대에 전달되기를 원한다. 그를 위해서는 오늘도 의 과대학 교수들은 불가사리가 되어야 한다.

Acknowledgements: None.

Funding: None.

Conflicts of interest: None.

\section{REFERENCES}

1. Na BJ, Lee K, Kim K, Song D, Hur Y. Experience of 
developing and implementing a motivation induction course for Konyang University Medical College freshmen. Korean J Med Educ 2012; 24: 141-152.

2. Ryue SH, Lee HB. Korean medical students' cognitive, emotional, and social characteristics. Korean J Med Educ 2012; 24: 103-115.

3. Ahn JH. The influence of gender on professionalism female in trainees. Korean J Med Educ 2012; 24: 153-162.
4. Kang BJ. Experience with using multiple types of visual educational tools during problem-based learning. Korean J Med Educ 2012; 24: 127-139.

5. Yoo HH, Park JH, Kim JS, Kim HT, Kim YJ, Kim DC. Analysis of the relationship between students' seating preferences and academic achievement in medical school. Korean J Med Educ 2012; 24: 117-125. 